\title{
Pulse height measurements and electron attachment in drift chambers operated with $\mathrm{Xe}, \mathrm{CO}_{2}$ mixtures
}

\author{
A. Andronic ${ }^{\mathrm{a}, 1}, \mathrm{H}$. Appelshäuser ${ }^{\mathrm{b}}$, C. Blume ${ }^{\mathrm{a}}$, \\ P. Braun-Munzinger ${ }^{a}$, D. Bucher ${ }^{c}$, O. Busch ${ }^{a}$, \\ A. Castillo Ramirez ${ }^{\text {a }}$, V. Cătănescu ${ }^{\text {d }}$, M. Ciobanu ${ }^{d}$, H. Daues ${ }^{a}$, \\ A. Devismes ${ }^{\text {a }}$, D. Emschermann ${ }^{\text {b }}$, O. Fateev ${ }^{\mathrm{e}}$, C. Garabatos $^{\mathrm{a}}$, \\ N. Herrmann ${ }^{b}$, M. Ivanov $^{a}$, T. Mahmoud ${ }^{\text {b }}$, T. Peitzmann ${ }^{\text {c }}$, \\ V. Petracek ${ }^{\mathrm{b}}$, M. Petrovici ${ }^{\mathrm{d}}$, K. Reygers ${ }^{\mathrm{c}}$, H. Sann ${ }^{\mathrm{a}}$, \\ R. Santo ${ }^{c}$, R. Schicker ${ }^{b}$, S. Sedykh ${ }^{\text {a }}$, S. Shimansky ${ }^{\text {e }}$, \\ R.S. Simon ${ }^{a}$, L. Smykov ${ }^{\text {e }}$, H.K. Soltveit ${ }^{\mathrm{b}}$, J. Stachel ${ }^{\mathrm{b}}$, \\ H. Stelzer ${ }^{a}$, G. Tsiledakis ${ }^{a}$, B. Vulpescu ${ }^{\text {b }}$, J.P. Wessels ${ }^{a}$, \\ B. Windelband ${ }^{\mathrm{b}}$, O. Winkelmann ${ }^{\mathrm{c}}, \mathrm{C} . \mathrm{Xu}{ }^{\mathrm{b}}, \mathrm{O}^{\mathrm{O}}$ Zaudtke $^{\mathrm{c}}$, \\ Yu. Zanevsky ${ }^{\mathrm{e}}, \mathrm{V}$. Yurevich ${ }^{\mathrm{c}}$ \\ ${ }^{a}$ Gesellschaft für Schwerionenforschung, Darmstadt, Germany \\ ${ }^{\mathrm{b}}$ Physikaliches Institut der Universität Heidelberg, Germany \\ ${ }^{\mathrm{c}}$ Institut für Kernphysik, Universität Münster, Germany \\ d NIPNE Bucharest, Romania \\ e JINR Dubna, Russia \\ for the ALICE Collaboration
}

\begin{abstract}
We present pulse height measurements in drift chambers operated with $\mathrm{Xe}, \mathrm{CO}_{2}$ gas mixtures. We investigate the attachment of primary electrons on oxygen and $\mathrm{SF}_{6}$ contaminants in the detection gas. The measurements are compared with simulations of properties of drifting electrons. We present two methods to check the gas quality: gas chromatography and ${ }^{55} \mathrm{Fe}$ pulse height measurements using monitor detectors.
\end{abstract}

Key words: drift chambers, $\mathrm{Xe}, \mathrm{CO}_{2}$ mixtures, electron attachment, gas chromatography

PACS: 29.40.Cs, 29.40.Gx 


\section{Introduction}

The ALICE Transition Radiation Detector (TRD) [1] has to provide both electron identification and particle tracking. To achieve this, accurate pulse height measurement in drift chambers operated with $\mathrm{Xe}, \mathrm{CO}_{2}(15 \%)$ gas mixture over the drift time of the order of $2 \mu \mathrm{s}$ (spanning $3 \mathrm{~cm}$ of drift length) is a necessary requirement. For such precision measurements, it is of particular importance not to loose charge by electron attachment, i.e. the absorption of drifting electrons by electronegative molecules present in the detector gas as contaminants. The large volume $\left(28 \mathrm{~m}^{3}\right)$ of the ALICE TRD and the high cost of xenon make the above arguments very serious for the operation of the final detector.

Attachment is a well studied and generally understood phenomenon, both fundamentally $[2,3]$ and concerning its practical implications for gas drift chambers [4]. For electron energies relevant to gaseous detectors (energies below a few eV), attachment occurs mainly via two mechanisms: resonance capture and dissociative capture. Resonance capture, also called Bloch-Bradbury mechanism [5], has the largest cross-section. It can be written as:

$$
\begin{aligned}
& I+e^{-} \rightarrow I^{-*} \\
& I^{-*}+S \rightarrow I^{-}+S^{*} .
\end{aligned}
$$

$I$ denotes the impurity and $S$ is a third body stabilizer, which in case of gas detectors, is usually the quencher. The star $(*)$ denotes a vibrationally excited state. Besides decaying by the resonant energy transfer $(2), I^{-*}$ could also decay by electron emission (autodetachment), in which case there is no signal loss. The rate of process (2), and thus the magnitude of attachment, depends on the concentration of $S$ and on the lifetime of the excited state $I^{-*}$. Also, as a result of different vibrational levels available for the energy transfer (2), the attachment depends on the type of quencher [4,6]. An excess of electron attachment with respect to the Bloch-Bradbury mechanism has been identified and assigned to van der Waals complexes [7].

The most common electronegative molecule is $\mathrm{O}_{2}$, present in gaseous detectors as a residual contaminant in the gas supply or from the atmosphere due to imperfect tightness of the system. Attachment on $\mathrm{O}_{2}$ has been extensively studied for Ar-based mixtures in ranges of parameters relevant for drift chambers [4,6]. Another common contaminant in gas detectors is $\mathrm{H}_{2} \mathrm{O}$, usually outgassed by assembly materials, and often appreciated as an ageing-limiting

1 Corresponding author: GSI, Planckstr. 1, 64291 Darmstadt, Germany; Email: A.Andronic@gsi.de; Phone: +49 615971 2769; Fax: +49 6159712989 
agent [8]. It has been found that, for certain Ar-based mixtures, attachment on $\mathrm{H}_{2} \mathrm{O}$ alone is negligible, but a few hundred ppm of $\mathrm{H}_{2} \mathrm{O}$ can double the attachment coefficient on $\mathrm{O}_{2}[4]$.

In one of our early measurements with TRD prototype chambers operated with $\mathrm{Xe}, \mathrm{CH}_{4}(10 \%)$ we observed electron attachment under very low contamination levels of $\mathrm{O}_{2}$ and $\mathrm{H}_{2} \mathrm{O}$. As that particular gas supply was exhausted during the measurements, we were not able to analyze it and so not able to attribute the attachment to a defined impurity. Subsequent measurements (performed with a new supply of Xe) proved to be free of visible attachment. However, recent observation of strong attachment, this time with the mixture $\mathrm{Xe}, \mathrm{CO}_{2}(15 \%)$ forced us to investigate the issue in more detail. We have been able to identify the impurity responsible for attachment: sulphur hexafluoride, $\mathrm{SF}_{6}$, which was found to be present at the ppm level in a xenon supply. This heavy gas, well known for its excellent insulating properties, has an extremely large cross section for electron attachment at low electron energies. We note that pulse height distributions as a function of drift time were measured before in drift chambers with Xe-based mixtures, also related to TRDs $[9,10,11,12]$. A decrease of the average pulse height as a function of drift time was observed in all these cases and it was attributed to electron attachment [12], but not quantitatively understood.

Here we report on measurements performed during prototype tests of the ALICE TRD [1]. Drift chambers operated with $\mathrm{Xe}, \mathrm{CO}_{2}$ mixtures are investigated. The experimental setup and method of data analysis are described in the next section. We then present measurements under clean conditions (no attachment). The following sections contain our measurements of attachment on oxygen and on $\mathrm{SF}_{6}$. We compare our results with simulations of charge transport for various gas mixtures. We present gas quality checks employing gas chromatograph analyses and ${ }^{55} \mathrm{Fe}$ measurements using especially-built monitoring detectors.

\section{Experimental setup}

Most of the results are obtained using prototype drift chambers (DC) with a construction similar to that anticipated for the final ALICE TRD [1], but with a smaller active area $\left(25 \times 32 \mathrm{~cm}^{2}\right)$. In Fig. 1 we present a schematic view of the detector. The DC has a drift region of $30 \mathrm{~mm}$ and an amplification region of $7 \mathrm{~mm}$. The anode wires (W-Au, $20 \mu \mathrm{m}$ diameter) have a pitch of $5 \mathrm{~mm}$. For the cathode wires ( $\mathrm{Cu}-\mathrm{Be}, 75 \mu \mathrm{m}$ diameter) we use a pitch of $2.5 \mathrm{~mm}$. We read out the signal on a segmented cathode plane. The pads (of $6 \mathrm{~cm}^{2}$ each) have either chevron [13] or rectangular shape. The entrance window $(25 \mu \mathrm{m}$ aluminized Kapton) simultaneously serves as gas barrier and as drift electrode. 


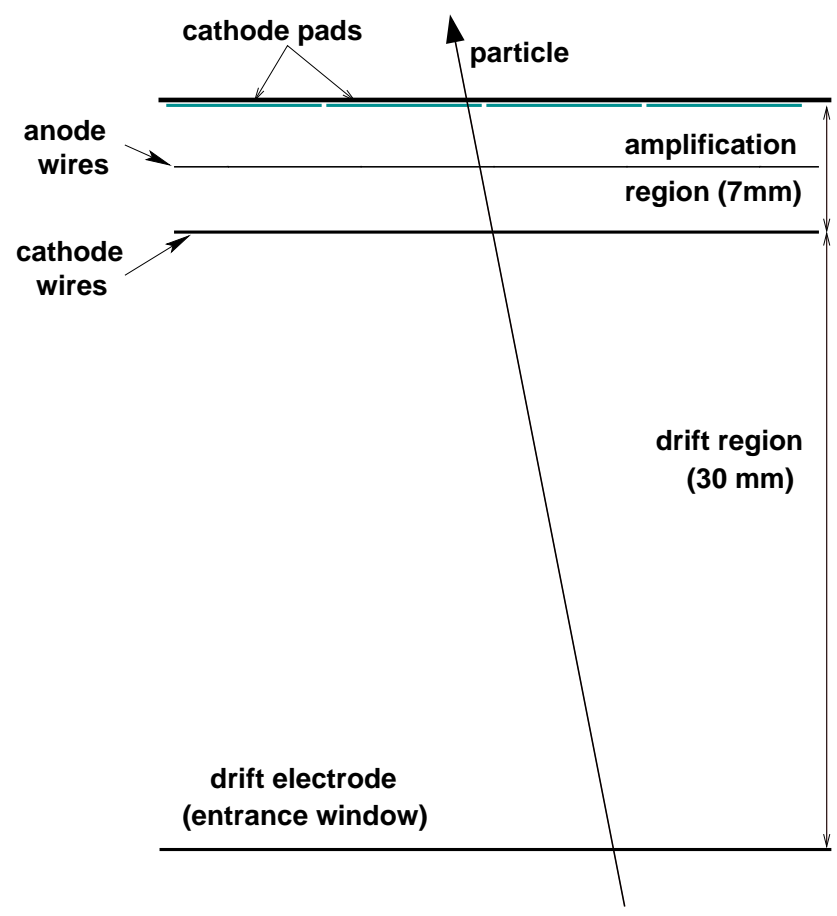

Fig. 1. Schematics of the drift chamber.

A charge-sensitive preamplifier/shaper (PASA) was especially designed and built (with discrete components) for the purpose of prototype tests. It has a gain of $2 \mathrm{mV} / \mathrm{fC}$ and noise of about 1800 electrons r.m.s. The FWHM of the output pulse is about $100 \mathrm{~ns}$ for an input step function. For the readout of the DC we use an 8-bit non-linear Flash ADC (FADC) system with $100 \mathrm{MHz}$ sampling frequency, $0.6 \mathrm{~V}$ voltage swing and adjustable baseline. The FADC sampling was rebinned in the off-line analysis in order to resemble the performance of the final detector [1]. The data acquisition (DAQ) is based on a VME event builder and was developed at GSI Darmstadt [14]. As the beam diameter is of the order of a few $\mathrm{cm}$, we usually limit the readout of the DC to 8 pads. This also minimizes data transfer on the VSB bus connecting the FADC and the event builder.

The measurements were carried out at beam momenta of $1 \mathrm{GeV} / \mathrm{c}$ at GSI Darmstadt [15] and $3 \mathrm{GeV} / \mathrm{c}$ at the CERN PS [16]. The beams were mixtures of electrons and negative pions. For the present analysis we have selected clean samples of pions using coincident upper thresholds on a Cherenkov detector and on a lead glass calorimeter (see ref. [17] for details). To minimize the effect of space charge on the pulse height measurement, which occurs for tracks at normal incidence to the anode wires (for which all charge collection takes place at a narrow spot on the anode wire), we adjusted the angle of incidence of the beam to about $15^{\circ}$ with respect to the normal incidence to the anode wires. A particle trajectory through the detector is sketched in Fig. 1.

The standard gas mixture for our detectors is $\mathrm{Xe}, \mathrm{CO}_{2}(15 \%)$ at 1 mbar above 
atmospheric pressure. The continuous flow of gas through the detectors is either vented out or recirculated via our gas system. The detectors are usually operated at gas gains around 8000. Our standard supply of xenon is from Messer-Griesheim [18] and proved to provide a very good detector performance. We have also used xenon from Linde [19], which provided a strikingly poor signal at first examination. $\mathrm{SF}_{6}$ was detected early on with gas chromatography techniques in this xenon supply, and its concentration was measured to be of the order of $1 \mathrm{ppm}$. Both xenon supplies were used in beam measurements reported on below.

\section{Measurements under clean conditions}

For these measurements we use $\mathrm{SF}_{6}$-free xenon. The oxygen content in the gas was continuously monitored and kept below $10 \mathrm{ppm}$ using a flow of 2-3 liters of fresh gas per hour into two chambers of about 9 liters total volume. The water content was about $150 \mathrm{ppm}$.

Distributions of average pulse height, $\langle P H\rangle$, as a function of drift time for different drift voltages are shown in Fig. 2 for pions of $1 \mathrm{GeV} / \mathrm{c}$ momentum. The detector gas is our standard mixture, $\mathrm{Xe}, \mathrm{CO}_{2}(15 \%)$. The time zero has been arbitrarily shifted by about $0.3 \mu$ s to have a measurement of the baseline and of noise. Similar distributions have been measured for the mixture $\mathrm{Xe}, \mathrm{CO}_{2}(20 \%)$.

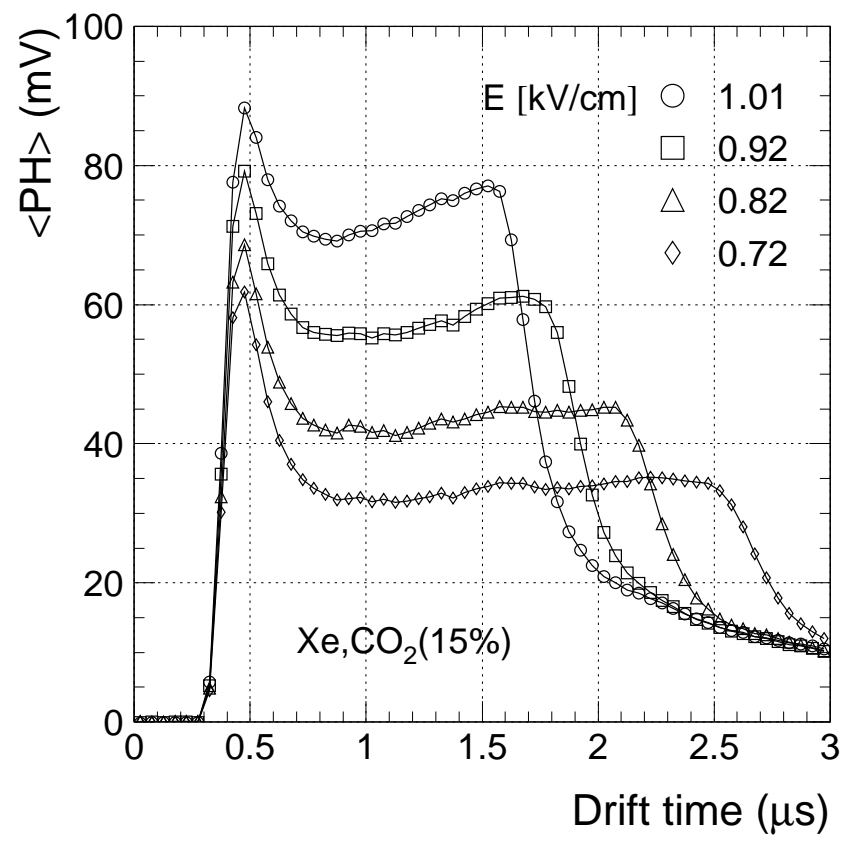

Fig. 2. The average pulse height as function of drift time for different drift fields for the mixture $\mathrm{Xe}, \mathrm{CO}_{2}(15 \%)$. 
The primary electrons from ionization energy loss of pions drift towards the anode wire, where they are amplified. The signal (charge induced on the pads) is determined mainly by the slow-moving ions, producing long tails in the PASA output. The overlap of these tails, convoluted with the response of the preamplifier, results in a slightly rising average pulse height as a function of the drift time, as seen in Fig. 2. The peak at short drift times originates from the primary clusters generated in the amplification region, where the ionization from both sides of the anode wires contributes to the same time interval. Note that, for the present conditions, lower values of drift field imply smaller drift velocity (see Fig. 7 in section 6), leading to a stretching of the signal over longer drift times. Our measurements established for the first time [17] the expected time evolution of the signal in drift chambers of the type studied here.

\section{Attachment on oxygen}

Again, for these measurements we use clean $\left(\mathrm{SF}_{6}\right.$-free) xenon. In Fig. 3 we show the average pulse height distributions as a function of drift time for different values of the oxygen content in the range of a few hundred ppm. A decrease of the signal as a function of drift time is seen when the concentration of oxygen increases. This is a clear indication of electron attachment. Notice that the signal in the amplification region, where electrons drift very little, is affected to a much smaller extent.

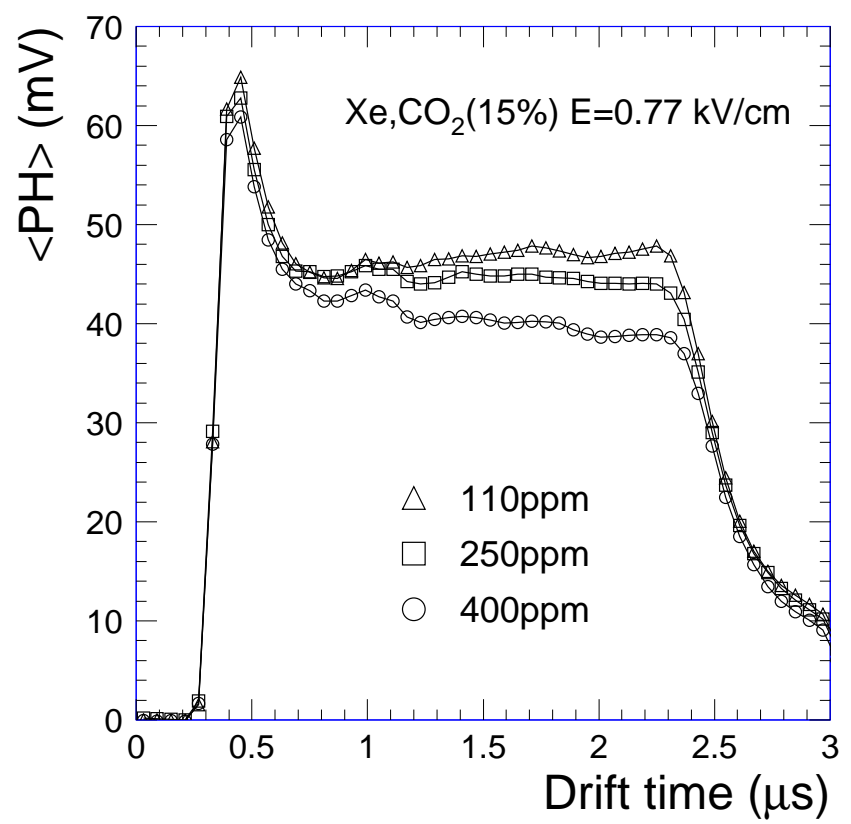

Fig. 3. Average pulse height as function of drift time for different values of the oxygen concentration in $\mathrm{Xe}, \mathrm{CO}_{2}(15 \%)$. 
In case of attachment, the number of electrons (and the corresponding measured pulse height) decreases exponentially as a function of drift time, $t$ :

$$
N(t)=N(0) \cdot \mathrm{e}^{-A \cdot t}
$$

where $A$ is the attachment rate [4]. At a given gas pressure, $p$ (which is the atmospheric pressure in our case), the attachment rate depends linearly on the concentration (partial pressure) of the impurity responsible for attachment, $p_{I}$, and can be factorized [4] as:

$$
A=p \cdot p_{I} \cdot C_{I}
$$

where $C_{I}$ is the attachment coefficient of the impurity $I$.

From the above measurements of pulse height distributions as a function of drift time we deduce, for the present value of the drift field of $0.77 \mathrm{kV} / \mathrm{cm}$, an attachment coefficient on $\mathrm{O}_{2}, C_{\mathrm{O}_{2}}=400 \mathrm{bar}^{-2} \mu \mathrm{s}^{-1}$. This value is very similar to values measured for $\mathrm{Ar}, \mathrm{CO}_{2}$ mixtures with comparable $\mathrm{CO}_{2}$ content [6] and more than an order or magnitude larger than values measured for $\mathrm{Ar}, \mathrm{CH}_{4}$ mixtures [4]. Given the short drift time in our detectors, attachment on oxygen does not impose any severe constraint on the tightness of the drift chambers for the final detector.

\section{Attachment on $\mathbf{S F}_{6}$}

All the measurements presented below have been carried out with pions of $3 \mathrm{GeV} / \mathrm{c}$ momentum, using Linde xenon with $1.1 \mathrm{ppm} \mathrm{SF}_{6}$ contamination. For these measurements the $\mathrm{O}_{2}$ and $\mathrm{H}_{2} \mathrm{O}$ contamination was $150 \mathrm{ppm}$ and $400 \mathrm{ppm}$, respectively. These rather large values arise because, due to the contamination of the xenon, the gas was vented and not recirculated (and, as a consequence, not filtered), as usual, through our gas system.

In Fig. 4 we show the average pulse height distributions as a function of drift time for different values of the drift field for the standard gas mixture, $\mathrm{Xe}, \mathrm{CO}_{2}(15 \%)$. Beyond the decrease of the pulse height due to longer drift times for lower drift fields, there is obviously a dramatic loss of signal due to electron attachment. The relative loss of signal is most pronounced between the two highest values of the drift field, for which the variation of the drift velocity is very small. At drift fields higher than $1 \mathrm{kV} / \mathrm{cm}$, the plateau of the average pulse height in the drift region starts to recover. This trend is explained by the fact that the energy of the drifting electrons increases with the electric field and, therefore, the attachment coefficient of the $\mathrm{SF}_{6}$-polluted 


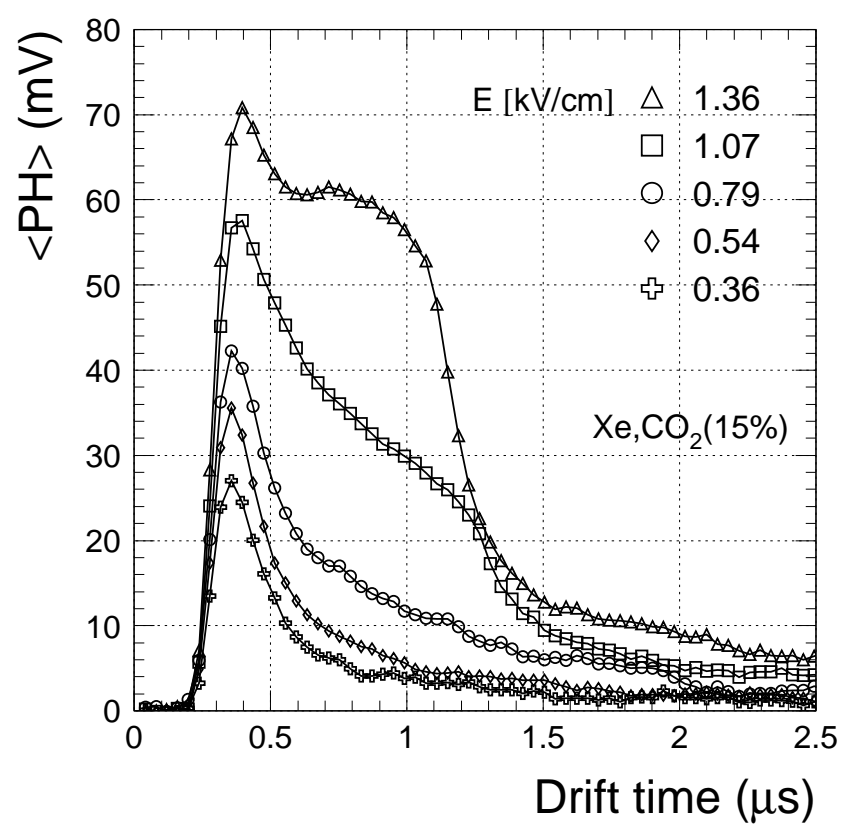

Fig. 4. Average pulse height as a function of drift time for different values of the drift field for the mixture $\mathrm{Xe}, \mathrm{CO}_{2}(15 \%)$.

mixture decreases (see next section). Similar behavior of the attachment as a function of drift field is known for Ar-based mixtures [4].

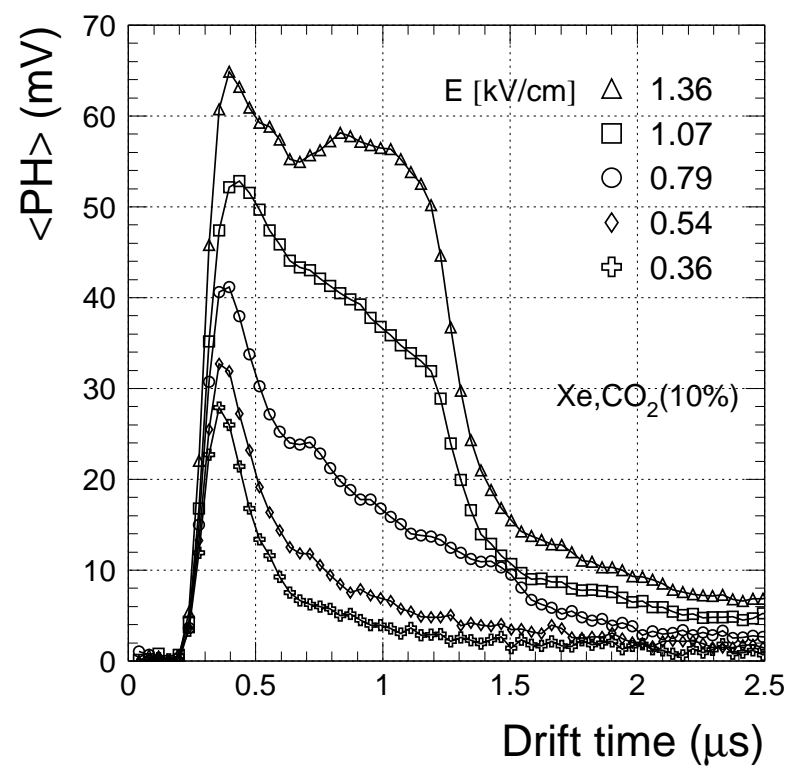

Fig. 5. As Fig. 4, but for the mixture $\mathrm{Xe}, \mathrm{CO}_{2}(10 \%)$.

The effect of different $\mathrm{CO}_{2}$ concentrations on the attachment efficiency of the contaminated xenon has also been investigated. Fig. 5 and Fig. 6 show the average pulse height distributions for $10 \%$ and $5 \% \mathrm{CO}_{2}$, respectively. The gas gains were not identical for the three concentrations of $\mathrm{CO}_{2}$, so the corresponding distributions can only be compared on a relative basis. Notice that, for the same drift field, the extension of the signal in time is different for dif- 


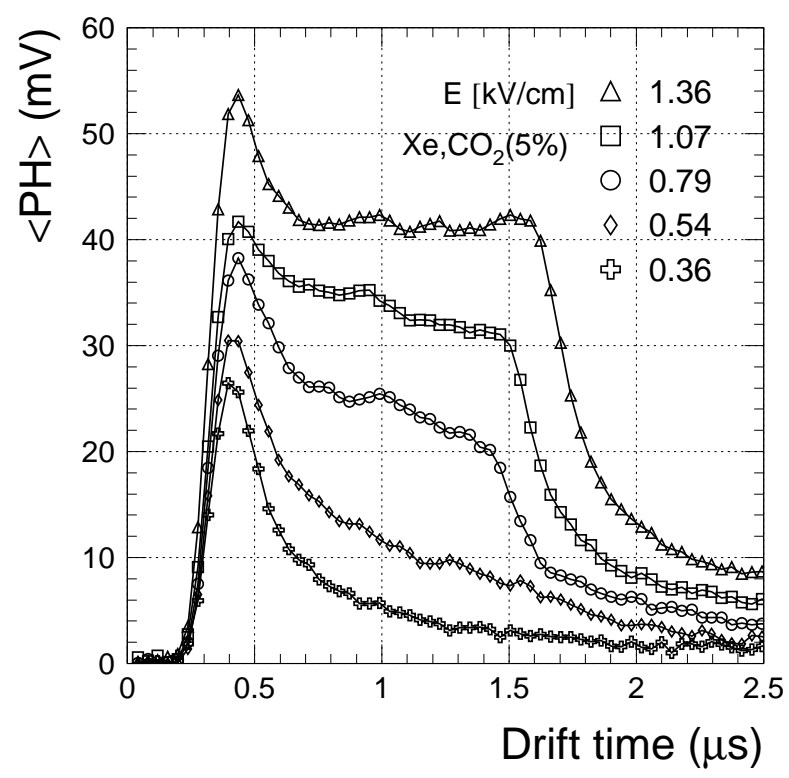

Fig. 6. As Fig. 4, but for the mixture $\mathrm{Xe}, \mathrm{CO}_{2}(5 \%)$.

ferent concentrations of $\mathrm{CO}_{2}$ because of different drift velocities (see Fig. 7 in the next section). The signal loss due to electron attachment decreases for lower $\mathrm{CO}_{2}$ concentrations. For the Xe, $\mathrm{CO}_{2}(5 \%)$ mixture, the pulse height distribution almost completely recovers at the highest electric field studied here. As we discuss in the next section, the variation of attachment as a function of quencher concentration is due to the dependence of the average energy of drifting electrons on the $\mathrm{CO}_{2}$ content.

\section{Comparison to calculations}

To understand the measurements presented above, we have performed calculations using the packages GARFIELD [20], MAGBOLTZ [21] and HEED [22]. In Fig. 7 we present the calculated drift velocities for 5\%,10\% and $15 \%$ of $\mathrm{CO}_{2}$ admixture in Xe. The dotted vertical lines mark the values of the electric field used for the measurements of attachment on $\mathrm{SF}_{6}$. The solid vertical line segments indicate the electric fields used for measurements under clean conditions (Fig. 2 in section 3). All these values are in a region where the drift velocity has a strong dependence on the drift field, a trend reflected in the measurements presented above.

To compare how well the measured pulse height distributions can be reproduced by simulations, we have calculated the detector signals using the GARFIELD package, under the exact experimental conditions. The preamplifier response has been included in these calculations. The time dependence of the average pulse height for different electric fields for the clean $\mathrm{Xe}, \mathrm{CO}_{2}(15 \%)$ 


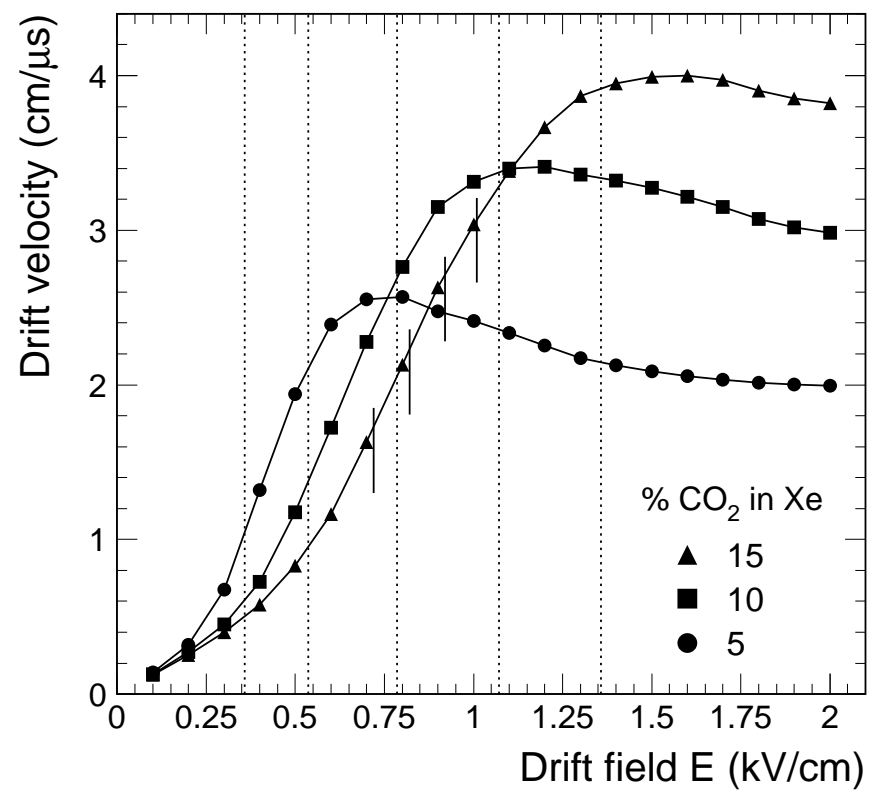

Fig. 7. Dependence of the drift velocity on the drift field for $5 \%, 10 \%$ and $15 \% \mathrm{CO}_{2}$ content in xenon, as calculated using GARFIELD/MAGBOLTZ [20,21].

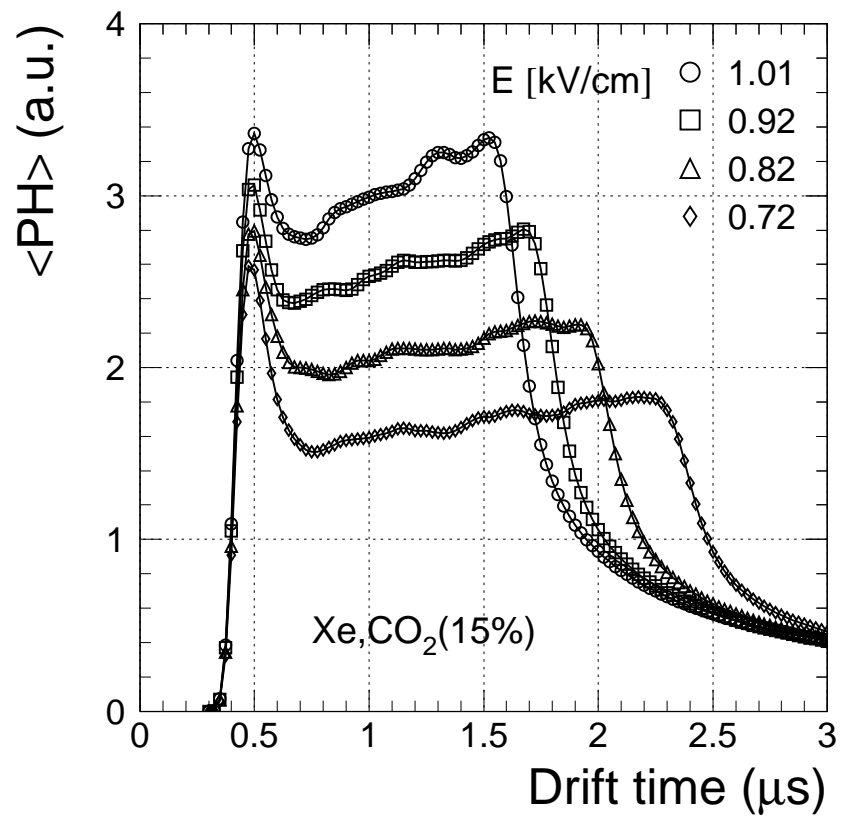

Fig. 8. Calculated average pulse height as a function of drift time for different values of the drift field for the mixture $\mathrm{Xe}, \mathrm{CO}_{2}(15 \%), \mathrm{SF}_{6}$-free.

mixture is presented in Fig. 8. For an easier comparison with the measurements (Fig. 2 in section 3), we introduced a time shift of $0.4 \mu$ s for these distributions. The calculations reproduce the measured signals reasonably well, although not in all details. The slope of signal increase in the drift region as a function of drift time is larger for the calculations. This may be an indication that some residual attachment is still present for the measured data, possibly as a result of oxygen, water and undetectectable amounts of other 
contaminants. The slightly asymmetric gaussian preamplifier response affects this slope very little. Already before folding the preamplifier response the calculated signals show a larger slope than the measured ones. For the lower values of the drift field the calculations are in disagreement with the measurement concerning the time extension of the signal. This discrepancy, reaching $14 \%$ for the field value of $0.72 \mathrm{kV} / \mathrm{cm}$, may reflect a different field dependence of the drift velocity in measurements and calculations. We note that a good agreement was found between calculations and measurements in other Xebased mixtures [23,24]. Since the bow of the entrance window (which is also the drift electrode) due to gas overpressure introduces an obvious uncertainty in the present measurements, it is too early to assess the above discrepacy quantitatively. Precision measurements of the drift velocity for the standard TRD gas mixture $\mathrm{Xe}, \mathrm{CO}_{2}(15 \%)$ are in progress.

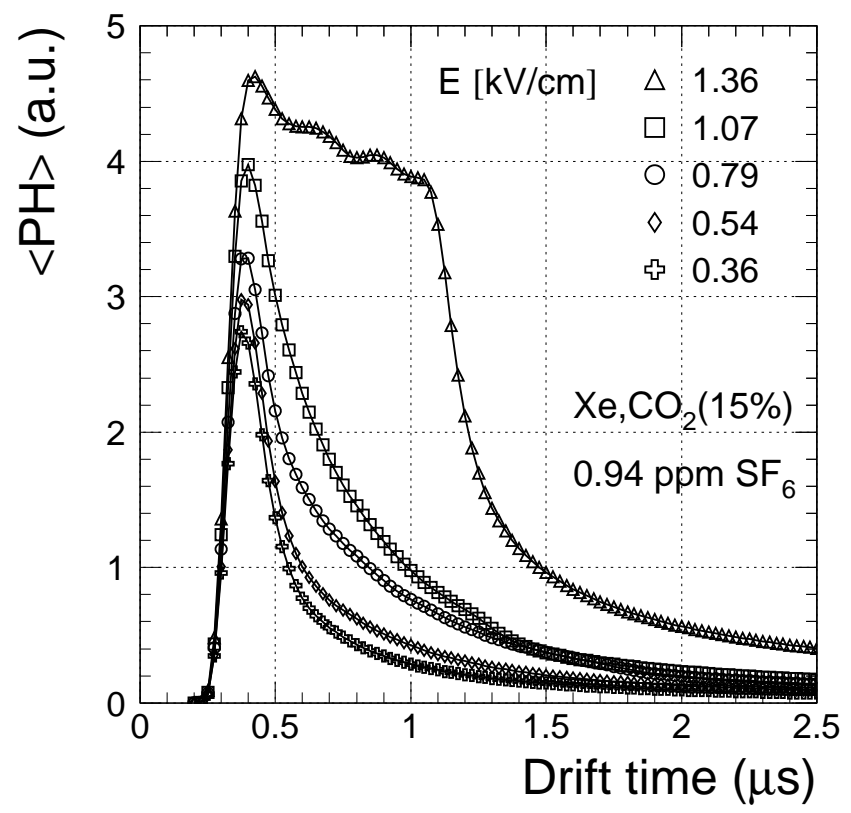

Fig. 9. Calculated average pulse height as a function of drift time for different values of the drift field for the mixture $\mathrm{Xe}, \mathrm{CO}_{2}(15 \%)$, with $0.94 \mathrm{ppm}$ of $\mathrm{SF}_{6}$ contamination.

Figure 9 shows the time dependence of the average pulse height for different electric fields for the $\mathrm{Xe}, \mathrm{CO}_{2}(15 \%)$ mixture with 0.94 ppm of $\mathrm{SF}_{6}$, corresponding to the $\mathrm{SF}_{6}$ fraction in the measurements. For a direct comparison with the measurements, the time shift is $0.3 \mu \mathrm{s}$ in this case. The measured attachment (Fig. 4 in the previous section) is reproduced almost quantitatively, except for the smoother reduction of attachment towards higher field values seen in the measurements. Not reproduced is the measured stronger variation of the signal in the amplification region.

We turn now to a more detailed investigation of the attachment on $\mathrm{SF}_{6}$. In particular, the measured drift field dependence of the attachment (nicely reproduced by simulations) is expected to reflect the characteristics of the attach- 


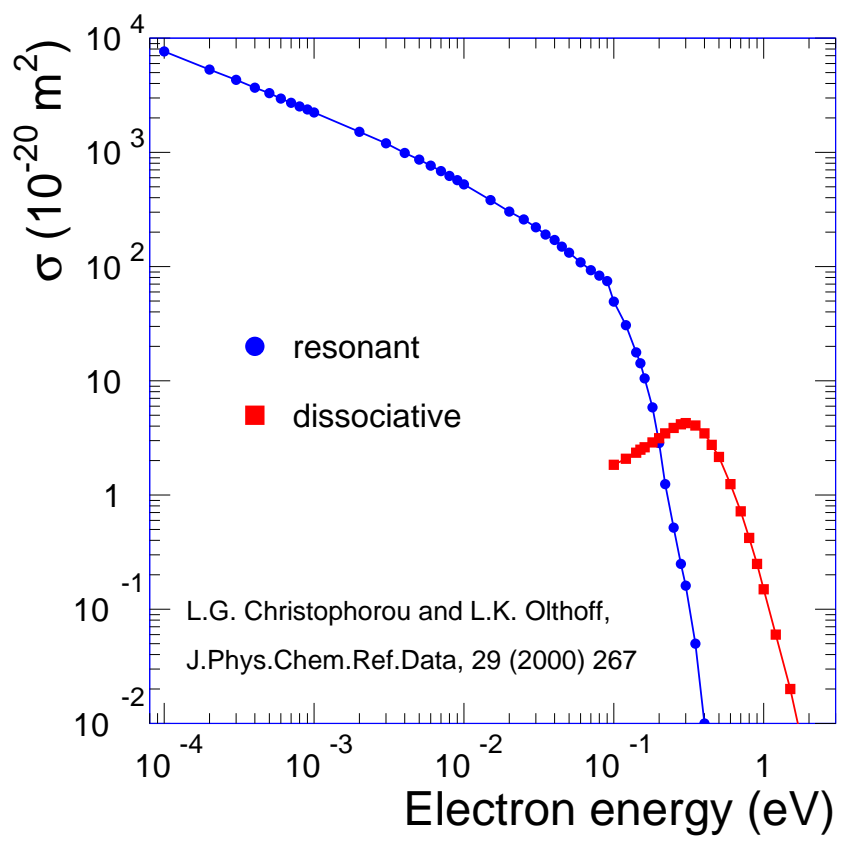

Fig. 10. The energy dependence of cross section of electron attachment on $\mathrm{SF}_{6}$. Recommended values from ref. [3] are plotted.

ment cross section as a function of electron energy. Indeed, the cross section of electron attachment on $\mathrm{SF}_{6}$ is large and has a strong energy dependence, as shown in in Fig. 10 [3]. Resonance capture, which leads to the formation of $\mathrm{SF}_{6}^{-}$, is the most important mechanism, with cross sections up to $8 \cdot 10^{-17} \mathrm{~m}^{2}$ for near-zero electron energies. The cross section of dissociative capture, resulting in a free fluorine atom and $\mathrm{SF}_{5}^{-}$, peaks at the value of $4 \cdot 10^{-20} \mathrm{~m}^{2}$ for electron energy of about $0.3 \mathrm{eV}$. These characteristics of attachment cross sections of $\mathrm{SF}_{6}$ are quite different compared to the $\mathrm{O}_{2}$ case [2]. For example, in case of $\mathrm{O}_{2}$, the cross section for dissociative capture peaks at about $10^{-22} \mathrm{~m}^{2}$ for electron energy of $6.5 \mathrm{eV}[2]$.

The energy spectra of the drifting electrons for various electric fields are shown in Fig. 11 for the mixture $\mathrm{Xe}, \mathrm{CO}_{2}(15 \%)$. These distributions are computed with the simulation program Imonte [21]. A significant high-energy component (energies above $1 \mathrm{eV}$ ) is present towards higher field values. The average energy of drifting electrons (also computed with Imonte), $\bar{\varepsilon}$, is plotted in Fig. 12 as a function of drift field for the three concentrations of $\mathrm{CO}_{2}$ in Xe. The average energy increases strongly with the electric field and is larger for smaller $\mathrm{CO}_{2}$ concentrations. The dotted vertical lines mark the values of the drift field used for the measurements. As the attachment cross section decreases as a function of the electron energy, the trends seen in Fig. 12 explain both the observed dependence of attachment on drift field and on $\mathrm{CO}_{2}$ concentration mixture.

The concentration of $\mathrm{CO}_{2}$ may influence the rate of the resonant energy transfer in the second step of the Bloch-Bradbury process (2), but this seems not to 


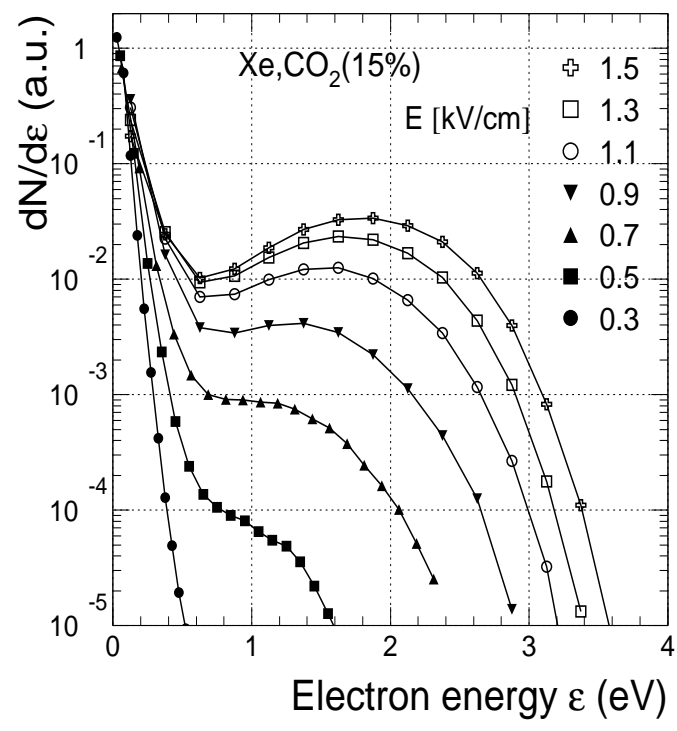

Fig. 11. Energy distribution of drifting electrons for different values of the drift field for the mixture $\mathrm{Xe}, \mathrm{CO}_{2}(15 \%)$.

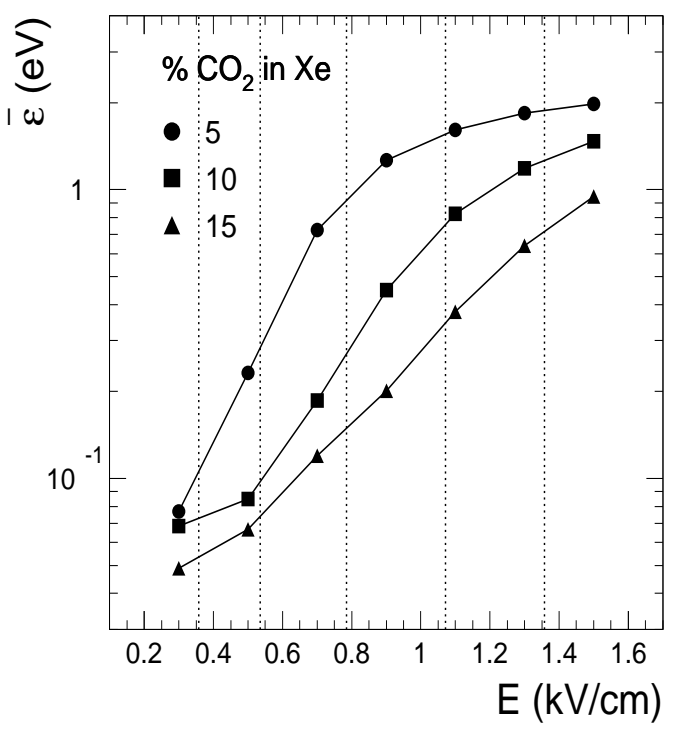

Fig. 12. Average energy of drifting electrons as a function of the drift field for different concentrations of $\mathrm{CO}_{2}$ in $\mathrm{Xe}$.

be the case for attachment on $\mathrm{SF}_{6}$. The measured pulse height distributions for $15 \%, 10 \%$ and $5 \% \mathrm{CO}_{2}$ (Figs. 4 to 6 in the previous section) for the field values of $1.36,1.07$ and $0.79 \mathrm{kV} / \mathrm{cm}$ (for which the corresponding average energies are similar, see Fig. 12), respectively, look very similar. Although we cannot make a quantitative statement, apparently all the influence of $\mathrm{CO}_{2}$ concentration on the attachment stems from the average energy of the drifting electrons. In contrast, a dependence of oxygen attachment on quencher content (beyond electron energy contribution) has been measured for $10 \%$ and $20 \% \mathrm{CH}_{4}$ in $\mathrm{Ar}$ [4]. This difference between $\mathrm{SF}_{6}$ and $\mathrm{O}_{2}$ attachment is probably the result of different lifetimes of the corresponding excited states of the negative ions. Indeed, the autodetachment lifetime is larger than $1 \mu \mathrm{s}$ for $\mathrm{SF}_{6}^{*-}[2]$, comparable with the drift time in our detectors, whereas for $\mathrm{O}_{2}^{*-}$ it is about $10^{-4} \mu \mathrm{s}$ [7].

\section{Methods for checking the gas quality}

Using xenon supplies as $\mathrm{SF}_{6}$-free as possible is an important requirement. In the following we describe our monitoring procedures, i.e. gas chromatography and ${ }^{55} \mathrm{Fe}$ pulse height measurements using dedicated monitor detectors.

Gas chromatography allows the detection and quantification of traces of pollutants in a gas, by separating the different species contained in the sampled gas in a chromatograph column, and by detecting them in a suitable detector placed downstream of the column. The separated effluents give rise to characteristic peaks in a time diagram which can be identified and quantified after 
proper calibration of the device. Since $\mathrm{SF}_{6}$ has a high electron capture cross section, an Electron Capture Device (ECD) is a suitable detector. An ECD consists of a cavity through which a so-called make-up gas (nitrogen) flows. Electrons from a beta source $\left({ }^{63} \mathrm{Ni}\right.$, maximum energy $\left.66 \mathrm{keV}\right)$ partly ionise the nitrogen gas. The total current produced is collected by an electrode. If an electronegative substance flows through the cavity at a given time, the missing collected charge is converted into a peak in the corresponding chromatogram. The area under the peak is proportional to the amount of electrons captured.

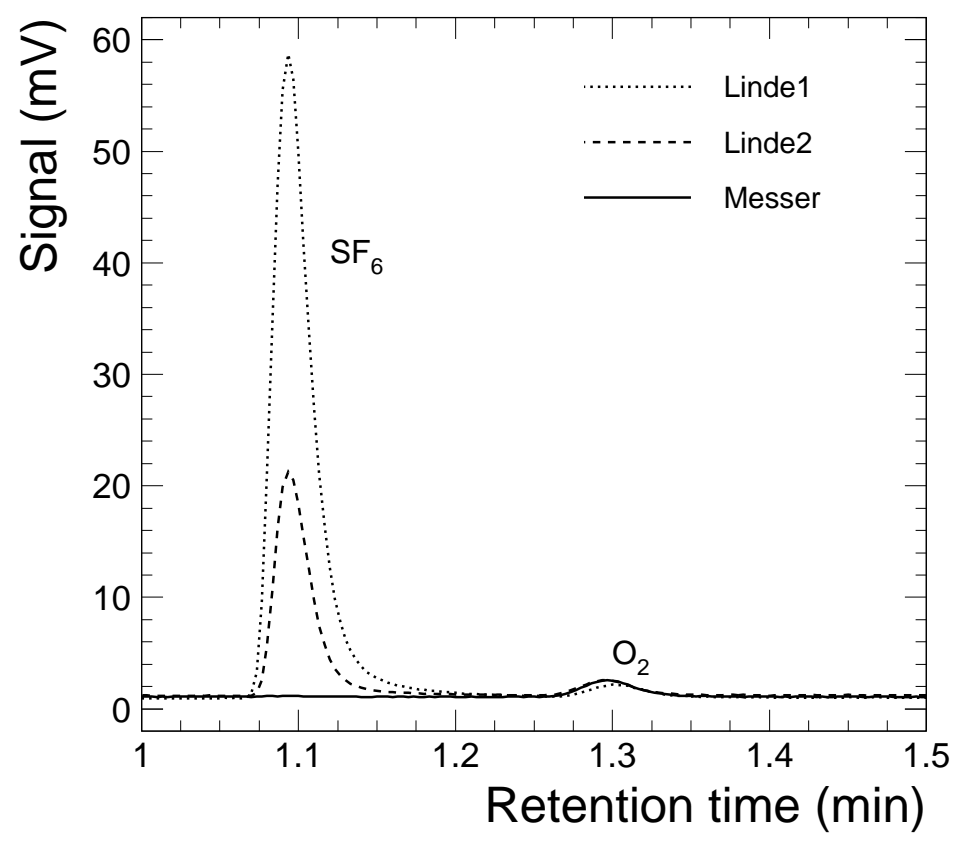

Fig. 13. Chromatograms of syringe-injected samples of three xenon supplies: $\mathrm{SF}_{6}$-free (Messer) and with $\mathrm{SF}_{6}$ contaminations of $0.3 \mathrm{ppm}$ (Linde2) and $1.1 \mathrm{ppm}$ (Linde1).

An ECD has been connected to our gas chromatograph [25] in order to analyse our different supplies of xenon. Concentrations as low as 1 ppb are detectable. The device has been calibrated for $\mathrm{SF}_{6}$ by putting small amounts of this gas into a glass container filled with helium. Special care has to be taken with the injection syringes since they get temporarily contaminated when exposed to high concentrations of $\mathrm{SF}_{6}$. In addition to the two xenon supplies (Messer and Linde1) used for the beam measurements reported above (section 5), we have also investigated a more recent supply from Linde (Linde2). The resulting chromatograms for the two kinds of xenon from Linde and the xenon from Messer-Griesheim are shown in Fig. 13. The calibration yields 1.1 and $0.35 \mathrm{ppm} \mathrm{SF}_{6}$ for the Linde1 and Linde2 gas samples, respectively, with an

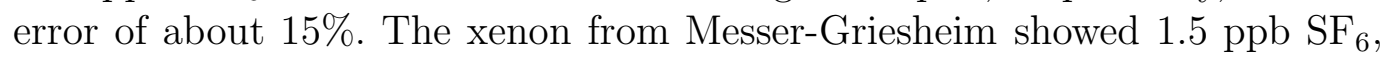
most probably coming from the contaminated syringe. The injection method also leads to inevitable air contamination, as revealed by the oxygen peak (corresponding to $400 \mathrm{ppm}$ ) in the chromatograms. 


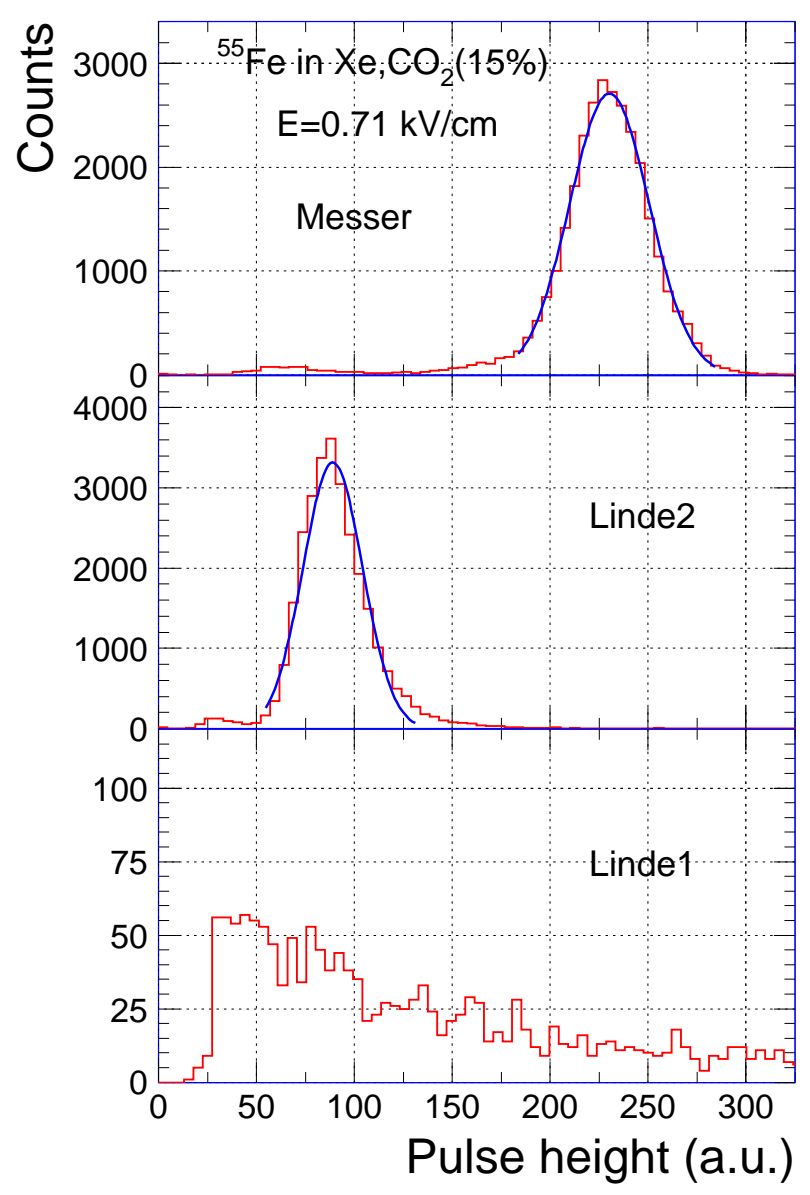

Fig. 14. Comparison of pulse height distributions of ${ }^{55} \mathrm{Fe}$ in $\mathrm{Xe}_{2} \mathrm{CO}_{2}(15 \%)$ for three supplies of xenon.

Gas chromatography is a simple, accurate and economic technique, but the chromatograph itself is a rather expensive device. However, for most detector applications, one does not necessarily need chromatography in order to assess the gas quality. Measurements with a ${ }^{55} \mathrm{Fe}$ source on standard detectors are sensitive enough to reveal possible problems due to attachment. In our case, in order to minimize Xe consumption for such tests, we have built small monitor detectors for the special purpose of checking the gas quality using ${ }^{55} \mathrm{Fe}$ pulse height measurements. These monitor detectors have the same electrical field configuration as the drift chambers used for the beam measurements described above. Their small volume of about half a liter minimizes the loss of xenon gas. A collimated ${ }^{55} \mathrm{Fe}$ source is placed in front of the entrance window. As the Xrays are absorbed preferentially at the beginning of the drift region, the cluster of primary electrons drifts in most cases $3 \mathrm{~cm}$ and is subject to attachment over this distance. For these measurements the anode voltage has been tuned for a gas gain of about $10^{4}$. Here we have used a different preamplifier, with a gain of $6 \mathrm{mV} / \mathrm{fC}$ and noise of about 1000 electrons r.m.s. As a result of a low flow through the detector, the contamination with $\mathrm{O}_{2}$ and $\mathrm{H}_{2} \mathrm{O}$ was 70 and 400 ppm, respectively. 
Figure 14 shows pulse height spectra for the three supplies of xenon discussed above. The value of the drift field (which corresponds roughly to the anticipated operational point of the final TRD in ALICE) is $0.71 \mathrm{kV} / \mathrm{cm}$. Compared to clean Xe (Messer), the $0.35 \mathrm{ppm} \mathrm{SF}_{6}$ contamination in Linde2 Xe leads to a pulse height distribution with a much smaller value for the main peak. In addition, a clear tail towards larger pulse height is seen, originating from absorption of X-rays deeper into the drift region, and thereby subject to less attachment loss. In case of Linde1 (1.1 ppm $\left.\mathrm{SF}_{6}\right)$ the ${ }^{55} \mathrm{Fe}$ signal is completely lost. The spectrum recorded is the result of $\mathrm{X}$-rays absorbed in the vicinity of the anode and of cosmic-ray background. Notice that the number of counts is much smaller in this case (for a comparable acquisition time). Notice also that, for the Messer and Linde2 cases, the escape peak of Xe (at $1.76 \mathrm{keV}$, compared to $5.96 \mathrm{keV}$ full energy of ${ }^{55} \mathrm{Fe}$ ) is clearly visible (in case of Linde2, the escape peak is partially cut by the threshold).

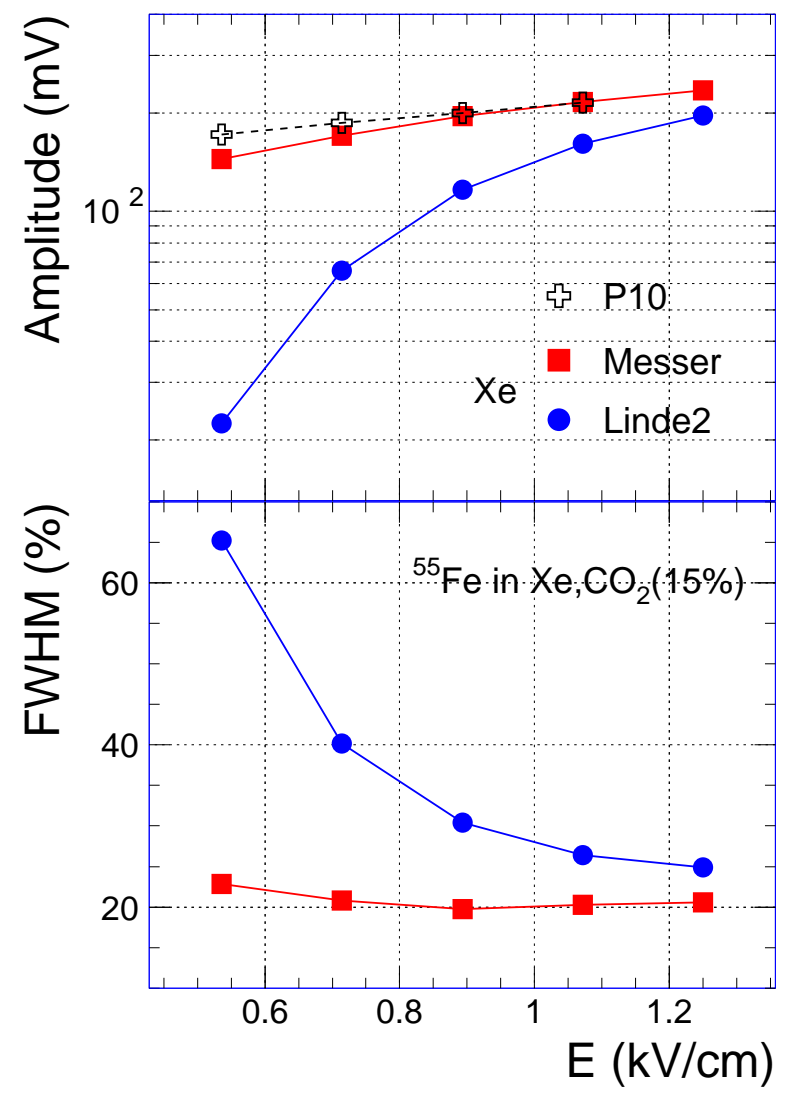

Fig. 15. Centroid (upper panel) and energy resolution (lower panel) of pulse height distributions of ${ }^{55} \mathrm{Fe}$ spectra in $\mathrm{Xe}, \mathrm{CO}_{2}(15 \%)$ as a function of drift field for two supplies of xenon.

When possible, we performed gaussian fits of the main peak (also plotted in Fig. 14) and extracted the centroid and energy resolution. Both quantities are influenced by attachment. In Fig. 15 we show the dependence of the amplitude of the main peak and its FWHM on the drift field for Messer and Linde2 supplies. The clean gas shows the expected variation of amplitude as a function 
of drift field arising from gain increase due to the transparency of the cathode wire grid. For comparison we include a measurement with an $\mathrm{Ar}, \mathrm{CH}_{4}(10 \%)$ mixture (P10). Compared to this, at low fields, even for clean Xe, small deviations are seen, consistent with attachment on $\mathrm{O}_{2}$; taking the P10 signal as reference, we deduce an attachment coefficient of $506 \mathrm{bar}^{-2} \mu \mathrm{s}^{-1}$ for the field value of $0.72 \mathrm{kV} / \mathrm{cm}$, in reasonable agreement to the value determined from beam tests (section 4). For the Linde2 case this dependence of the signal on electric field is much stronger, as a result of the attachment. This is confirmed by the energy resolution (lower panel in Fig. 15), which improves dramatically when going (as a function of drift field) from strong to weak attachment.

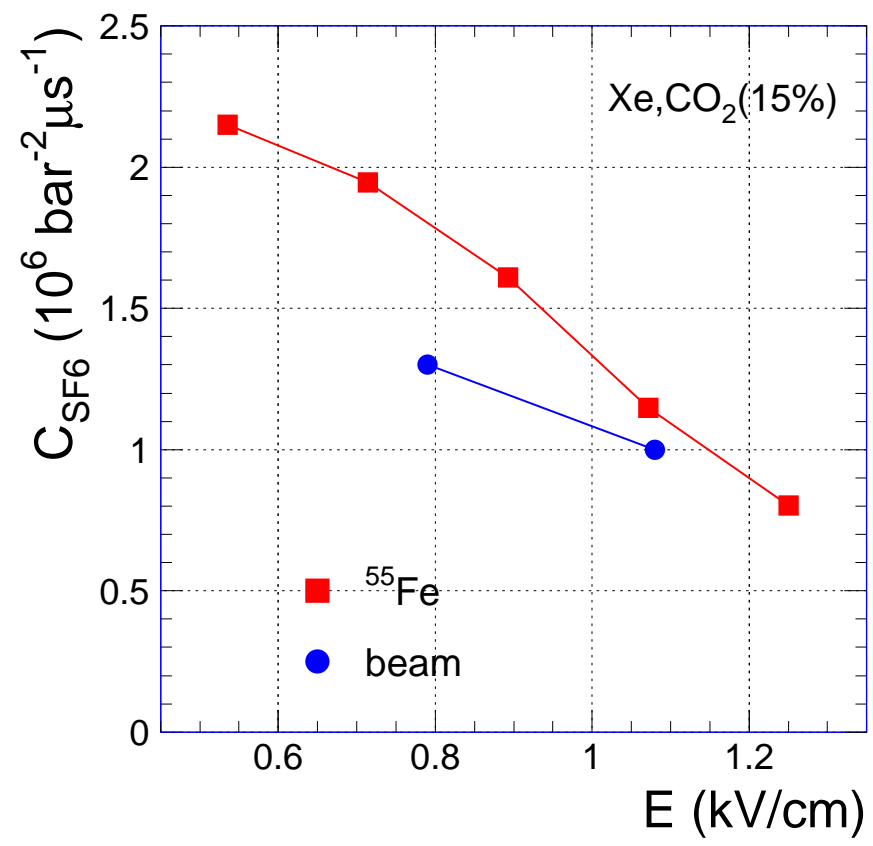

Fig. 16. Attachment coefficient on $\mathrm{SF}_{6}$ in $\mathrm{Xe}, \mathrm{CO}_{2}(15 \%)$ as a function of drift field.

We have determined the attachment coefficient on $\mathrm{SF}_{6}$ (under our experimental conditions mentioned above) using the ratio of the ${ }^{55} \mathrm{Fe}$ amplitudes for the Messer and Linde2 cases. The drift time is extracted using the drift velocities calculated with GARFIELD and assuming that the absorption of X-rays takes place right at the entrance window. The results are presented in Fig. 16 as a function of drift field. As expected, the attachment coefficient on $\mathrm{SF}_{6}$ is large, of the order of $10^{6} \mathrm{bar}^{-2} \mu \mathrm{s}^{-1}$. In addition, it has a pronounced dependence on the drift field, in line with our arguments presented in section 6. If we consider the absorption length of $5.9 \mathrm{keV}$ X-rays in the $\mathrm{Xe}, \mathrm{CO}_{2}(15 \%) \mathrm{mix}$ ture, which is about $3 \mathrm{~mm}$, the attachment coefficient would increase by $10 \%$. For comparison, we include in this plot the results obtained from the beam mesurements, taking as a reference the time distribution of signals measured in the clean case and normalized in the amplification region. Taking into account the uncertainties, in particular coming from the normalization for the beam measurements, the extracted values for the attachment coefficient are in reasonable agreement. 


\section{Summary}

We have performed measurements of pulse height distributions in drift chambers operated with $\mathrm{Xe}, \mathrm{CO}_{2}$ mixtures. After studying the general behavior of these distribution under clean conditions, we have investigated the role of oxygen and $\mathrm{SF}_{6}$ contamination of the detection gas. A small signal loss due to attachment is seen for $\mathrm{O}_{2}$ impurities up to a few hundred ppm. In case of $\mathrm{SF}_{6}$, a contamination even at the level below 1 ppm produces a dramatic loss of signal over our drift length of about $3 \mathrm{~cm}$. Attachment on $\mathrm{SF}_{6}$ is studied here for the first time concerning its practical implications for gas detectors. As the $\mathrm{SF}_{6}$ was found accidentally in some xenon supplies, it is important to have a careful monitoring of the $\mathrm{SF}_{6}$ contamination when precision measurements are performed using Xe-based gas mixtures in drift chambers. We have used ECD gas chromatography analysis to detect and quantify small traces of $\mathrm{SF}_{6}$. We have shown that measurements of ${ }^{55} \mathrm{Fe}$ signals in monitor detectors are very sensitive to $\mathrm{SF}_{6}$ contamination, thus allowing an inexpensive in situ check of the gas quality.

\section{Acknowledgments}

We acknowledge the skills and dedication of A. Radu and J. Hehner in building our detectors. We are indebted to S. Ilie and C. Jeanpetit for the first analysis of our gas. We appreciate the advice from R. Veenhof concerning the GARFIELD calculations. We acknowledge P. Szymanski for help during the measurements at CERN.

\section{References}

[1] ALICE TRD Technical Design Report, CERN/LHCC 2001-021, October 2001; http://www.gsi.de/ alice/trdtdr.

[2] L.G. Christophorou, Atomic and Molecular Radiation Physics, WileyInterscience, 1971 (Chapter 6).

[3] L.G. Christophorou and J.K. Olthoff, J. Phys. Chem. Ref. Data 29 (2000) 267.

[4] M. Huk, P. Igo-Kemenes and A. Wagner, Nucl. Instr. Meth. Phys. Res. A 267 (1988) 107.

[5] F. Bloch and N.E. Bradbury, Phys. Rev. 48 (1935) 689.

[6] S. Wenig, R. Bock, H.G. Fischer, R. Sendelbach and R. Stock, GSI Scientific Report 1993, 94-01, p. 264 (1994). 
[7] Y. Kokaku, Y. Hatano, H. Shimamori and R.W. Fessenden, J. Chem. Phys. 71, (1979) 4883.

[8] J.A. Kadyk, Nucl. Instr. Meth. Phys. Res. A 300 (1991) 436.

[9] Y. Watase et al., Nucl. Instr. Meth. Phys. Res. A 248 (1986) 379.

[10] R.D. Appuhn, K. Heinloth, E. Lange, R. Oedingen and A. Schlösser, Nucl. Instr. Meth. Phys. Res. A 263 (1988) 309.

[11] M. Holder and H. Suhr, Nucl. Instr. Meth. Phys. Res. A 263 (1988) 319.

[12] J.-F. Detoeuf et al., Nucl. Instr. Meth. Phys. Res. A 265 (1988) 157.

[13] B. Yu et al., IEEE Trans. Nucl. Sci. vol. 38 (1991) 454.

[14] H.G. Essel and N. Kurz, IEEE Trans. Nucl. Sci. vol. 47 (2000) 337.

[15] J. Diaz et al., Nucl. Instr. Meth. Phys. Res. A 478 (2002) 511.

[16] CERN PS, http://psdoc.web.cern.ch/PSdoc/acc/pscomplex.html.

[17] A. Andronic et al., IEEE Trans. Nucl. Sci. vol. 48 (2001) 1259 nucl-ex/0102017.

[18] Messer-Griesheim, http://www.messer.de/

[19] Linde AG, http://www.linde.de/

[20] R. Veenhof, Nucl. Instr. Meth. Phys. Res. A 419 (1998) 726; http://consult.cern.ch/writeup/garfield/.

[21] S.F. Biagi, Nucl. Instr. Meth. Phys. Res. A 421 (1999) 234.

[22] I. Smirnov, HEED, an ionization loss simulation program, 1997.

[23] T. Kunst, B. Götz and B. Schmidt, Nucl. Instr. Meth. Phys. Res. A 324 (1993) 127; B. Schmidt, private communication.

[24] U. Becker et al., Nucl. Instr. Meth. Phys. Res. A 421 (1999) 54.

[25] Thermo Finnigan, http://www.thermo.com/. 